\title{
Alter
}

Revue de phénoménologie

22 | 2014

Hans Jonas

\section{Clonons un être humain}

Jacques Dewitte

\section{OpenEdition}

Journals

Édition électronique

URL : http://journals.openedition.org/alter/313

DOI : $10.4000 /$ alter.313

ISSN : 2558-7927

\section{Éditeur :}

Association ALTER, Archives Husserl (CNRS-UMR 8547)

\section{Édition imprimée}

Date de publication : 15 novembre 2014

Pagination : 211-232

ISBN : 978-2-9550449-0-2

ISSN : 1249-8947

\section{Référence électronique}

Jacques Dewitte, «Clonons un être humain », Alter [En ligne], 22 | 2014, mis en ligne le 01 décembre 2017, consulté le 10 décembre 2020. URL : http://journals.openedition.org/alter/313 ; DOI : https:// doi.org/10.4000/alter.313

Ce document a été généré automatiquement le 10 décembre 2020.

Revue Alter 


\title{
Clonons un être humain
}

\author{
Jacques Dewitte
}

\section{NOTE DE L'AUTEUR}

Version remaniée d'une conférence donnée au colloque « Hans Jonas. Une éthique de la vie », à l'Université de Paris Panthéon-Sorbonne le 26 février 2011.

\section{Préambule}

1 Le principe responsabilité avait été conçu en deux parties : une partie «systématique » et une partie « casuistique » La première partie a paru sous ce titre en 1979. Pour la seconde, il publia en 1985 Technik, Medizin und Ethik. Zur Praxis des Prinzips Verantwortung², qui, en lieu et place du projet initial, impossible à réaliser en raison de son grand âge ${ }^{3}$, consistait en un ensemble d'études partielles parues de 1969 à 1984 portant sur des thèmes tels que l'art médical, le clonage humain, le rôle créateur de l'homme par rapport à son propre patrimoine génétique, la question de la mort cérébrale, les questions posées par les techniques d'assistance à la vie. Pourtant, malgré le caractère fragmentaire et inachevé de ces études, on est frappé par leur lucidité et leur caractère visionnaire. Jonas a pris position en particulier sur la question de la mort cérébrale (en 1970) ${ }^{4}$ et sur celle du clonage (en 1974) et à une époque où elles ne ne se posaient pas avec tant d'acuité qu'aujourd'hui.

2 La tâche de cette nouvelle casuistique est énorme. Par où commencer pour aborder ce «large spectre technologique ", se demande Jonas dans l'avant-propos du recueil ? Au lieu de faire ce qu'on aurait pu attendre de lui : partir de la menace de la destruction de l'humanité par la bombe atomique (problème central chez Günther Anders) ou des ravages de l'environnement naturel, il croit préférable, étant donné que nous ne disposons pas d'une "science intégrale de l'environnement", de commencer par le domaine de la biologie humaine. « Le mieux est de partir de ce qui nous est le plus proche, là où la technique prend directement l'homme pour objet, et où notre connaissance de 
nous-mêmes [...] peut lui répondre directement, donc le domaine de la biologie humaine et de la médecine » (TME, 9 et 11).

Ce choix est-il seulement le résultat d'une évaluation relative à la conjoncture où il se trouve? Je suis enclin à penser que cela révèle quelque chose d'essentiel quant à son propos. Quoi qu'il en pense et en dise lui-même, l'objet principal de la réflexion de Jonas n'est pas vraiment la menace pesant sur la nature et la vie organique, mais bien une menace concernant l'humanité de l'homme. Provenant non pas d'un danger extérieur, mais d'un projet consistant à réaliser un homme nouveau, une humanité parfaite. L'idée fondamentale qui sous-tend toute sa pensée est que l'amélioration de l'homme au moyen de la technique pourrait menacer la survie même de l'humanité. Deux finalités sont en conflit : les espoirs portant sur les améliorations futures de la condition humaine peuvent en effet compromettre la préservation d'une humanité authentique (qui, comme il l'écrit contre Bloch, est là « depuis toujours » et non pas à venir), l'impératif catégorique de la perpétuation de l'humanité imposant ainsi une limite (impossible à établir a priori) à ce que Jonas appelle le méliorisme.

\section{Le clonage}

Le texte Biological Engineering - A Preview a paru en 1974 dans les Philosophical Essays et remonte à un exposé oral présenté à l'Université de Chicago (sans doute autour de 1969)5. A Preview: un aperçu, une avant-première, un tour d'horizon. Mais aussi une prémonition. Aujourd'hui, on est frappé par l'extraordinaire préscience, par la capacité visionnaire de Jonas. Or, ce texte n'est jamais cité, ni en France ni en Allemagne, alors qu'on se réfère souvent à Habermas qui est intervenu plus tard dans cette discussion. Le titre allemand de la version de 1982 parue dans la revue Scheidewege - Lasst uns einen Menschen klonieren (clonons un être humain), entre-temps réédité dans Technik, Medizin und Ethik - est une paraphrase de la phrase divine : «Lasst uns den Menschen machen nach unserem Abbild» (faisons l'homme à notre image), ce qui indique bien que l'enjeu est profondément métaphysique.

5 Comme d'autres textes de ce recueil, il ne s'agit pas d'un texte systématique et bien poli. C'est le fruit d'une réflexion encore ouverte. On constate - c'est une caractéristique de toute l'œuvre de Jonas - des différences entre la version américaine, parue en 1974, et la version allemande, parue en 1982, ces différences témoignant d'une pensée en train de se faire ${ }^{6}$.

6 En outre, il s'avère très souvent que la version allemande est plus riche et plus dense que la version anglaise. D'où, bien que mes traductions se réfèrent principalement à la version allemande, mon effort pour tenir compte des deux versions et pour proposer des fragments de traduction qui tiennent compte des deux à la fois ${ }^{7}$.

7 L'article commence par des considérations générales et didactiques en examinant d'abord l'eugénisme ancien avant d'aborder le domaine nouveau, dit «futuriste». Il se termine par une présentation assez brève des techniques de chirurgie génétique, de travail sur l'ADN. C'est la partie médiane, dans les deux versions, qui est consacrée au clonage, envisagé sous deux angles: un examen de la «duplication de l'excellence», puis une « critique existentielle du clonage ». Je présenterai ces deux aspects dans l'ordre inverse. Mais il faut retenir que, parmi les différentes finalités possibles du projet de clonage 
humain, sera retenue par Jonas la plus «noble»: cloner un être exceptionnel afin de réitérer son unicité même.

\section{La critique de Leon $\mathrm{R}$. Kass}

8 La méditation de Jonas sur le clonage s'est développée dans le cadre d'un dialogue avec le philosophe américain Leon R. Kass 8 . Leur première rencontre remonte à l'automne de 1969, lorsque Kass a organisé un symposium sur les questions éthiques du progrès médical. Les années suivantes, ils sont devenus collègues au Hastings Center. Ces deux penseurs juifs ont en commun une référence déclarée à l'héritage biblique et à l'idée de l'homme créé à l'image de Dieu. Mais cette même référence débouche, concernant le projet du clonage, sur deux arguments critiques assez différents.

Kass place au centre de sa réflexion ${ }^{9}$ l'idée judéo-chrétienne de la « dignité de chaque être humain particulier » qui existe « dans une relation égale au Créateur ». C'est cette dignité qui serait violée par le clonage, ce qui est illustré au moyen d'une belle métaphore provenant du Midrash du Talmud (dans la version anglaise de 1974, Jonas ne la cite que dans la note, mais il la mettra en avant dans la version allemande de 1984):

«Un homme frappe beaucoup de pièces de monnaie d'une seule forme et elles sont toutes identiques les unes aux autres, mais le roi qui est au-dessus de tous les rois façonne chaque homme à l'image du premier homme et pourtant aucun n'est identique à l'autre ${ }^{10}$

Est clairement précisée la différence entre le modèle d'un artisan-démiurge qui façonne des objets sur le même moule, et le modèle d'une création dans laquelle apparaissent des individus à chaque fois différents et pourtant tous «à l'image de Dieu ». L'idée est très belle: alors qu'un artisan humain frappe des médailles certes individuelles par leur matérialité mais identiques quant à leur forme, le roi qui est au-dessus des rois, c'est-àdire Dieu, façonne chaque homme comme étant à l'image du premier homme (et donc à sa propre image) alors qu'aucun n'est identique à un autre. Il faut donc admettre, même si ce n'est pas explicité, que l'Ebenbildlichkeit, la relation à une « image de Dieu » existe, mais dans une modalité différente. Ce n'est pas une imitation de la forme, mais plutôt de la liberté qui a présidé à la création première.

11 Cette référence de nature métaphysique et religieuse permet à Kass de formuler, à l'encontre du projet du clonage, cet impératif éthique, exprimé en un «droit » : le droit à l'unicité du génotype. Chaque individu a droit à un génotype différent, singulier. Il est interdit de lui donner un génotype qui aurait déjà existé : « Est-ce que cela n’a pas un sens de dire que chaque personne a un droit naturel à ce qu'on ne lui refuse pas délibérément un génotype unique? », demande Kass. "Si un tel droit existe », commente Jonas, " alors son refus serait déjà condamnable dans le cas des jumeaux monozygotiques délibérément produits, [...] et a fortiori dans le cas du clonage » (PhE 159).

\section{Jonas reprend la main}

12 Jonas reprend partiellement à son compte l'argument de Kass, tout en introduisant une autre perspective: il met au centre de l'attention non pas l'identité ou la différence objective, mais la situation subjective du supposé clone humain, ce qui l'amène à souligner, non l'unicité du génotype, mais celle de l'existence. 
Tout en ayant une grande sympathie pour l'idée sous-jacente, je n'en ferai pas mon argument. Car s'il y a un droit à l'unicité, il s'agit de l'unicité de l'existence (being) dont l'unicité du génotype peut être ou non une condition nécessaire; nous n'en savons tout simplement rien. (Pour ma part, je ne le crois pas; en fait, le double cloné de quelqu'un d'autre sera, à cet égard, tout à fait unique à sa manière, mais sur un mode probablement misérable !) (PhE, 160) génotype, mis en avant par Kass) à un point de vue subjectif et intérieur. Il imagine (en un acte d'imagination empathique), quelle sera la situation d'un sujet qui saurait d'une manière ou d'une autre (éventuellement par des «fuites ») qu'il est la duplication d'un individu ayant déjà existé et ayant déjà fait ses preuves dans l'existence, puisque, par définition, il s'agit de quelqu'un d'éminent et de célèbre (je reviendrai plus loin sur cet aspect).

16 Est restituée ainsi une dimension fondamentale de la condition humaine : le fait que l'être humain se pose d'une manière ou d'une autre la question qui suis-je? (comme l'écrivait Augustin, l'homme est « une question pour lui-même »), une interrogation qui n'est pas statique, face à un miroir ou à un interlocuteur supposé savoir ce que lui-même ne sait pas, mais ouverte et qui sous-tend le cours même de l'existence. Or, cette interrogation, cette quête de soi-même, est évidemment liée à la surprise que chacun est non seulement pour les autres, mais pour lui-même. Avec quelque chose d'attirant et d'excitant, mais éventuellement aussi d'angoissant (comme pour toutes les modalités de la liberté).

Dans la situation jusqu'ici imaginaire que représente le clonage, mais qui pourrait devenir une réalité effective, la vie individuelle se réduirait en principe à un da capo, à la réitération d'une vie déjà existante, déjà accomplie, ayant déjà déployé toutes ses potentialités. Elle se verrait privée de cette incertitude et de la part d'ouverture qui lui est inhérente : de la surprise que toute existence est pour elle-même. Jonas écrit en ce sens : 
Le point décisif est que le génotype engendré sexuellement est un novum en soi, inconnu de tous au commencement et qui, au porteur non moins qu'aux autres, doit encore se révéler (sich offenbaren) dans l'accomplissement de l'existence. L'ignorance est ici une condition préalable de la liberté ; le nouveau coup de dés, une fois jeté, doit se découvrir lui-même dans l'effort sans guide de vivre sa vie pour la première et unique fois, c'est-à-dire de devenir un Soi dans la rencontre avec un monde qui est aussi peu préparé au nouveau venu que celui-ci ne l'est à lui-même. (TME 188, et PhE 159)

C'est du Jonas à l'état pur: on trouve dans ce passage son ton et ses thèmes caractéristiques: ceux du "coup de dés", et donc d'une forme de hasard ou de contingence féconde, et du "nouveau», du surgissement de quelque chose ou de quelqu'un qui n'est pas dérivable des états antérieurs - ce qui est aussi l'un des thèmes centraux de la pensée de Hannah Arendt : l'idée de la naissance et de la natalité.

\section{Le droit à l'ignorance}

19 C'est par rapport à cette idée de l'existence humaine (comme autre chose que le simple fait d'« exister ») que le clonage apparait comme une privation et une monstruosité. Car si ce projet devait se réaliser, un être humain naîtrait et commencerait à vivre et à exister en ayant non seulement une identité préétablie, mais surtout un savoir préalable de sa propre identité. Il serait donc privé de cet aspect. Le défi constitué par l'anticipation d'une telle situation fait ressortir une dimension jusque là inaperçue de la vie humaine : l'importance d'une forme particulière d'incertitude, de secret ou d'ignorance, d'une opacité à soi qui s'avère être la condition même de l'expérience de soi et de la surprise de l'existence, car, encore une fois, un être qui surgit dans le monde n'est pas seulement nouveau pour les autres, desquels il doit être accueilli comme l'est le nourrisson, mais pour lui-même. L'existence n'est pas le simple accomplissement d'un projet pré-existant, mais un déploiement tâtonnant s'effectuant dans le temps. Le projet de «cloner un être humain » fait donc ressortir a contrario une dimension humaine fondamentale inaperçue jusque là, et dont on prend peu à peu conscience.

Ainsi l'aventure (Wagnis) de la vie est-elle privée de son ouverture attirante mais aussi angoissante. On a permis au passé d'anticiper sur l'avenir par un savoir inauthentique sur ce dernier, dans la plus intime de toutes les sphères, celle de la question «qui suis-je? » Cette question doit venir du secret et ne peut trouver sa réponse que si la recherche de celle-ci demeure accompagnée du secret. Le secret, condition de l'interrogation et de la recherche, est même, pour celui qui cherche la réponse, la condition de possibilité de ce qui, peut-être, deviendra alors la réponse. La divulgation (offenbarsein) inauthentique au commencement, l'absence subjective du secret, détruit cette condition de la croissance authentique. Peu importe que le savoir supposé soit vrai ou faux (et il y a de bonnes raisons de supposer qu'il est essentiellement faux en soi); il est funeste pour l'acquisition de sa propre identité. Car ce qui est important existentiellement, c'est ce que la personne clonée pense est obligée de penser - qu'elle est, et non pas ce qu'elle « est » objectivement, au sens substantiel de l'être. Bref, le produit du clonage est privé à l'avance de la liberté , qui ne peut s'épanouir que sous la condition de l'ignorance. Priver délibérément un être humain à venir de cette liberté est un crime inexpiable qui ne peut être commis ne fût-ce qu'une seule fois. (TME, 191-192, PhE, 162)

Cela conduit à la formulation d'un nouvel impératif : le droit de la vie humaine à rester une surprise pour elle-même, qui implique une ignorance dans un sens bien précis: l'ignorance initiale quant à ce que l'on est, au « qui » singulier qui se révèlera dans son 
existence et ses actes eux-mêmes. À la place du droit à l'unicité du génotype avancé par Leon Kass, Jonas avance un autre droit: le droit à l'ignorance de ce que l'on « est» (ou « doit être ») et donc la nécessité de formuler un nouvel « impératif catégorique »:

Le commandement éthique [...] est donc [...] : respecte le droit de toute vie humaine à trouver sa propre voie et à être une surprise pour elle-même. À la question de savoir comment cette défense d'une ignorance quant à soi-même est compatible avec le vieux commandement du "connais-toi toi-même", il n'est guère difficile de répondre. Il suffit de comprendre que la découverte de soi que ce commandement nous impose est elle-même un chemin du devenir de ce Soi (eben dieses Selbst); à partir du donné inconnu, il se produit lui-même (bringt sich hervor) en même temps qu'il fait connaissance avec lui-même (seinem Sich-Bekannt-werden), ce qui a lieu dans les épreuves de la vie et serait obturé par ce savoir préalable. (TME, 194, PhE, 163)

21 C'est un passage très dense, typique de la prose philosophique de Jonas, marqué par un style qui fait songer à la fois à Hegel et à Heidegger ${ }^{11}$. Y a-t-il incompatibilité entre l'exigence philosophique et rationaliste traditionnelle: "connais-toi toi-même " modalité du pathos de la connaissance - et la découverte d'un «droit à l'ignorance » ? N'y a-t-il pas incompatibilité entre l'idéal des Lumières et un tel constat obscurantiste ? Entre Lumières et obscurité, voire obscurantisme? Non, répond Jonas, mais on doit admettre une part d'opacité logée dans l'exigence même de la lucidité et de la transparence.

Récapitulons. La critique de Jonas s'est articulée en trois moments successifs : 1. la mise en évidence de la nouveauté, de la découverte de soi et de la "surprise pour soi-même » comme dimension de l'existence humaine. 2. La perte de cette dimension avec le projet du clonage. 3. L'énonciation d'un nouvel impératif éthique, d'un droit inhérent à l'humanité de l'homme inaperçu jusque-là.

\section{Existentialisme et essentialisme}

Cette "critique existentielle» du clonage pourrait être appelé aussi «critique existentialiste ». L'un des traits constants de la pensée jonassienne est une inspiration existentialiste, qui n'exclut pas, sur d'autres sujets, un essentialisme ${ }^{12}$. Telle est, en effet, l'inspiration profonde et la grandeur de l'existentialisme, qu'il soit heideggérien ou sartrien, que Jonas reprend à son compte : un pathos de la liberté, de l'ouverture, de l'indétermination et du choix. L'être humain existe - et même ek-siste - comme cet être qui, soit n'a pas d'identité préétablie, soit ne détient pas préalablement un savoir quant à cette identité (je laisse cette question en suspens). La première éventualité, plus radicale, est celle de la pensée sartrienne, la seconde celle de la pensée jonassienne. L'être humain est - Sartre et Heidegger se rejoignent - cet être pour qui « il en va dans son être de son être », un être qui, originairement, se rapporte à lui-même sans se saisir.

La critique existentielle du clonage met en évidence - comme la monstruosité à prévenir - une distorsion de la temporalité, que l'on peut caractériser comme un « vol du futur ». Les générations futures - les générations vivantes - seront privées de leur présent et asservies aux générations mortes. Cette critique existentielle pourrait s'appeler aussi une « critique temporelle » du clonage.

Une autre version du nouvel impératif pourrait être : «Agis de telle sorte que l'existence individuelle demeure un présent véritable tourné vers un avenir inconnu qui ne se réduise pas à être la répétition d'une existence passée ». Contre un aplatissement de la 
temporalité, il importe de préserver la dimension initiale et initiatique de toute existence, ce qui en elle est à la fois une initialité et une initiation en train de se faire. "Il a été autorisé au passé d'anticiper (vorgreifen) le futur par un savoir inauthentique sur celuici ». C'est en cela que consiste ce vol perpétré envers les générations futures de leur présent, bref l'asservissement des vivants aux morts.

Le contrôle immanent de l'homme sur sa propre nature générique (Artnatur) apparait comme le couronnement triomphant de ce pouvoir. [...] Mais un pouvoir de qui, et sur qui ou sur quoi ? Manifestement le pouvoir de ceux de maintenant sur ceux de demain, qui seront les objets sans défense des décisions [...] des planificateurs d'aujourd'hui. Le revers du pouvoir actuel est la servitude ultérieure des vivants vis-à-vis des morts. [...] Quoi qu'ils fassent (ou souhaitent), cela ne fera qu'exécuter la loi que le pouvoir dominateur a imposée sur leur genèse (Entstehung). (PhE, 145 sq.)

Donc, l'analyse critique du clonage humain - en tant que projet - débouche donc sur l'énonciation d'un droit fondamental inaperçu jusque là, un «droit à l'ignorance » qui prend la forme de cet impératif : « respecte le droit de toute vie humaine à trouver sa propre voie et à être une surprise pour elle-même" (TME, 194). Or, ce droit-là, nous ne le connaissions pas auparavant; certains progrès technologiques et les menaces potentielles qu'ils comportent pour l'humanité de l'homme nous l'ont fait découvrir pour la première fois. Cela ne veut pas dire que nous l'ayons simplement inventé ou créé, mais que nous apercevons pour la première fois avec clarté une dimension qui sans doute existait déjà, mais sans que nous en ayons été conscients.

[...] nous devons faire ce pas au vu d'une situation entièrement nouvelle et encore hypothétique qui représente effectivement la première occasion pour l'activation d'un droit qui jusqu'ici avait sommeillé de manière latente faute de pouvoir être appliqué. (TME 190, PhE 161)

C'est aussi une nouvelle manifestation de l'esprit existentialiste qui imprègne une bonne part de la pensée de Jonas. Nous ne détenons pas un concept préalable de l'homme, une "essence qui précèderait l'existence" comme disait Sartre. Ce concept se révèle à l'épreuve d'une confrontation au réel donnée dans une menace. C'est à l'occasion de celleci que ce qui était jusque-là "assoupi» se révèle clairement. Bien sûr, philosophiquement, le statut de cet «ensommeillement» est fort délicat. Peut-on supposer ou postuler un tel état d'ensommeillement et de latence?

28 À la différence de l'homme sartrien, l'homme jonassien n'est pas un sujet qui se fait, se façonne ou se choisit librement. C'est plutôt un sujet qui se découvre avec étonnement, et qui, de manière tout de même classique, se connaît lui-même. Mais cette connaissance n'est pas la simple découverte factuelle d'une essence prédéterminée, déposée quelque part (en quelque « armoire » transcendantale ${ }^{13}$ ) et que l'on trouverait seulement. Il faut comprendre que cette connaissance est aussi, en quelque manière, un faire ou un agir, le résultat d'une démarche active. Mais justement - c'est là le nœud et la dimension abyssale - nous ne pouvons pas savoir exactement quelle est en nous-mêmes, dans notre vie, la part exacte du connaître et du faire, du donné préalable et de la transformation intérieure.

La pensée de Jonas est une pensée de la contingence (il insiste beaucoup sur la dimension du hasard, recourt fréquemment à l'image du jeu de dés), mais c'est une contingence en quelque sorte réflexive. Le sujet n'est pas seulement jeté dans le monde. C'est une contingence primairement affectée de réflexivité, d'une auto-interrogation : qui suis-je ? 
Mais ce n'est pas la simple découverte de ce qu'on serait de toute façon, puisqu'elle va de pair avec une action et une révélation de potentialités inédites.

Il faut avouer que c'est aussi, par là, une pensée difficile : peut-on, sans contradiction, être à la fois existentialiste et essentialiste? On ne peut même pas parler ici d'une structure d'après-coup : il serait inexact d'avancer que se révèlerait après coup ce qui était déjà préétabli. Pour Jonas, il s'agit d'une découverte qui est aussi une invention (sans que l'on puisse distinguer l'une de l'autre). L'« herméneutique du Dasein » heideggérienne devient chez Jonas une véritable « heuristique du Dasein » : je me découvre moi-même au fil de ma propre expérience, c'est-à-dire aussi de ma confrontation avec le monde (et les autres). Ce n'est pas une simple introspection: le Dasein s'interprète lui-même originairement mais, ce faisant, il se découvre lui-même, il s'apparaît à lui-même pour la première fois. $\mathrm{Ce}$ " pour la première fois » est un mot-clé quasiment magique.

Prenons un peu de recul. La conception de Jonas est belle, mais elle a aussi, si on l'examine de près, quelque chose de vertigineux. Est-ce qu'une telle conception - qui se tient sur le fil du rasoir - est tenable ? Elle pose une foule de problèmes. Peut-on soutenir l'idée d'une ignorance quant à soi-même, et l'expérience d'une auto-découverte sans tomber dans l'attitude d'une pure indétermination vide, ou dans la "résolution" heideggérienne qui n'a pas de contenu, ou avant tout comme contenu la résolution ellemême ? Ou bien encore : peut-on soutenir que l'essence ou l'identité de quelqu'un serait non seulement une découverte, mais un faire précédé d'aucun concept préexistant ou d'aucun inné ? Comment allier la dimension de la « découverte » avec celle du « faire »?

Ou, pour le dire autrement, qu'en est-il de toute la dimension de l'« inné » ? C'est-à-dire de tout ce qui, chez l'être humain, est préformé biologiquement ou génétiquement? On ne peut pas imaginer en faire table rase pour laisser se déployer un pur "projet" existentiel. Là-dessus, la position de Jonas semble être la suivante : l'important est que nous n'avons pas accès à un savoir sur cet «inné »; il se peut que la découverte de soi effectuée au cours de l'existence soit en partie la réalisation de tendances innées et préexistantes (il ne le nie pas). Mais elles se réalisent de toute façon d'une manière qui n'est pas le simple résultat de déterminations.

\section{L'heuristique de la crainte}

Il y a donc un redoublement de l'inspiration existentialiste de Jonas. Il s'agissait d'abord de l'examen de la situation existentielle où chaque homme, dans son histoire individuelle unique, est et doit rester « une surprise pour lui-même ». Il s'agit maintenant de la portée de cette leçon pour notre compréhension de l'essence de l'homme (et des « droits de l'homme »). Dans les deux cas, il n'y a pas de savoir préalable ni, en tout cas, de concept déterminé et exhaustif de l'homme; il y a une indétermination de ce que nous pouvons savoir sur l'homme.

34 La critique existentielle du clonage est un bel exemple de ce que Jonas a appelé l'«heuristique de la crainte» (Heuristik der Furcht) et je m'étonne qu'il ne le dise pas explicitement. Je rappelle les passages du Principe responsabilité où cette notion est introduite et définie. Elle consiste à renoncer, au moins en partie, à l'idée que nous détiendrions déjà un concept préalable de l'homme ou de la nature humaine. Non pas en ce sens que l'idée même d'une nature humaine soit une illusion ou une construction, mais en ce sens que, compte tenu des mutations technologiques et anthropologiques effectives 
ou imminentes du monde contemporain, nous pouvons prendre conscience pour la première fois d'aspects de cette nature (ou de ce concept) qui, jusqu'à présent, étaient passés inaperçus parce qu'ils allaient de soi. Bref, nous appréhendons (au sens d'une connaissance) pour la première fois ce concept lorsque nous appréhendons (au sens d'une crainte) qu'il est menacé :

C'est seulement la distorsion anticipée de l'homme qui nous procure le concept de l'homme qu'il s'agit de préserver [...]. Contrairement à toute logique et à toute méthode, le savoir à ce sujet procède de ce contre quoi il faut se protéger. [...] Nous savons seulement (erst) ce qui est en jeu lorsque nous savons que cela est en jeu ${ }^{14}$.

La prise de conscience d'un "droit à l'ignorance ", révélée par la potentialité du clonage, en est un nouvel exemple: nous savons seulement, ou mieux: pour la première fois (l'adverbe allemand "erst", pivot verbal de la pensée de Jonas, a ce double contenu) qu'une forme d'ignorance est essentielle depuis que cette dimension est menacée par le projet du clonage humain. Nous découvrons ainsi une nouvelle facette inaperçue de l'image de l'homme.

Le moment est venu de m'étendre sur un problème de traduction. Dans mon article princeps de $1988^{15}$, j'avais traduit tout naturellement Heuristik der Furcht par « heuristique de la crainte ». Dans sa traduction française parue en 1992, Jean Greisch a traduit par «heuristique de la peur", formule reprise par tous les commentateurs. L'idée même de Jonas a suscité une controverse ; on lui a reproché de se rapprocher de Hobbes, ce qui est un malentendu concernant ses intentions philosophiques. Car même s'il parle dans ce contexte d'un effroi (Erschrecken), Jonas n'a pas employé le mot Angst - peur, angoisse mais Furcht, crainte pour quelque chose. Les mots n'ont certes pas un sens absolument fixé à l'avance, mais ici la différence semble assez claire. Les deux termes semblent quasiment synonymes, mais avec une nuance imperceptible et pourtant décisive (dans le contexte de l'éthique jonassienne).

Angst désigne une peur à la fois énorme et indéterminée : c'est l'angoisse au sens que Heidegger a donné à cette notion, une peur sans objet précis, ou dont l'objet est global, étant la survie elle-même. Furcht, la crainte, en revanche, désigne un sentiment qui ne porte pas seulement sur la cause du danger, mais sur quelque chose qui est menacé : on craint pour quelque chose, on tremble pour sa vie, pour sa place, pour sa dignité, etc. La crainte ainsi comprise n'est donc pas globale; elle a un contenu. C'est une Furcht um etwas , un « fürchten um », une notion intentionnelle au même titre que la conscience, l'amour ou la haine, qui portent sur un objet extérieur au sentiment lui-même.

Tel est bien le sens de cette « heuristique » à travers la menace, on découvre l'objet même qui est menacé, pour lequel on a lieu de craindre. La traduction française publiée, sans être un contresens, infléchit la pensée de Jonas dans un sens hobbesien, elle favorise le malentendu et empêche de comprendre le sens profond de cette heuristique : découvrir pour la première fois, à travers la menace, la valeur et le sens même de ce qui est menacé, découvrir de nouveaux aspects de l'humanité de l'homme. On peut se demander d'ailleurs pourquoi il faudrait parler d'une "heuristique » si on comprenait ce mot au sens d'un simple « choc salutaire " qui nous fait réfléchir (au sens du "catastrophisme éclairé » défendu par Jean-Pierre Dupuy).

La notion de "crainte » ne doit pas être comprise comme simplement affolée et encore moins comme angoissée; elle implique un amour de certains objets dont nous ne soupçonnions pas que nous y tenions à ce point (notre réflexion devrait « consulter nos craintes préalablement à nos désirs, afin de déterminer ce qui nous tient réellement à 
cœur » (PV, 64, PR, 49 sq.) et dont nous découvrons qu'ils sont menacés. Trembler pour quelque chose qui nous tient à cœur ; c'est le sens de l'heuristique de la crainte).

Le choix du traducteur du terme de " peur » au lieu de celui de "crainte » pour rendre Furcht dans l'expression Heuristik der Furcht fut, non pas certes un contre-sens, mais une décision malheureuse, une maladresse qui a favorisé une interprétation erronée. Le vocable «peur » a donné le ton. L'ouvrage a été accueilli comme s'il avait été écrit dans une tonalité de peur majeure qui n'est pas vraiment la sienne.

\section{Excursus philologique}

41 Outre les réserves que je viens d'exprimer sur le choix initial, je constate que la traduction comporte, à propos du concept de « Furcht», de nombreuses incohérences. Au chapitre 2, Furcht est traduit uniformément par « peur ». Mais dans le développement où Jonas oppose «wünschen » et «fürchten» (PV 64) - d'une part, ce que nous souhaitons, d'autre part ce pour quoi nous craignons - "fürchten» est rendu fort justement par "craindre ", de même que dans la note 1 de ce chapitre, attenante à ce paragraphe («mais lorsque je dois craindre pour la santé de mon enfant, parce que subitement il y a des raisons de craindre, alors je le sais » PR 50). Dans ce passage, le traducteur a donc adopté le parti qui me paraît le meilleur, mais comme une exception par rapport à l'ensemble, et avec une autre incohérence : au milieu d'un développement consacré à « nos craintes » (PR 49), on retrouve la formule de l'« heuristique de la peur » (PR 50).

$42 \mathrm{Au}$ chapitre 6, dans le paragraphe de préambule, où Jonas précise ses intentions fondamentales, c'est-à-dire explicite le sens même du titre de son ouvrage, il écrit : «Dem Prinzip Hoffnung stellen wir das Prinzip Verantwortung gegenüber, nicht das Prinzip Furcht », ce qui est traduit par: «Au principe espérance, nous opposons le principe responsabilité et non le principe crainte » (PV 390, PR 300). « Das Prinzip Furcht» est donc traduit par «le principe crainte ", ce qui revient à adopter la solution qui a ma préférence : rendre " Furcht " par «crainte». Mais dans tout ce qui suit, le traducteur retourne à la solution retenue au chapitre 2 et traduit « Furcht » par « peur ». L'important paragraphe intitulé « Furcht, Hoffnung und Verantwortung» (PV 391) devient donc: «La peur, l'espérance, la responsabilité » (PR 300).

Une page plus loin, dans le développement de ce paragraphe où Jonas avance le concept de «selbstlose Furcht» (PV 392), nouveau revirement: l'expression est traduite par " crainte désintéressée " (PR 301) - de même que "Furcht und Zittern » est traduit, très justement, par "crainte et tremblement». Mais dans la note complémentaire où Jonas oppose à la "selbstlose Furcht ", attitude qu'il défend, la "selbstische Furcht » hobbesienne (PV 392), c'est-à-dire oppose une crainte pour quelque chose d'autre à une crainte pour soimême - ces deux termes sont traduits respectivement par "peur désintéressée » et par "peur égoïste» (PR 301). De sorte que, dans le même paragraphe, la même formule : « selbstlose Furcht » est rendue une première fois par « crainte désintéressée » et la seconde fois par " peur désintéressée ». (Sur ce point précis, le traducteur aurait pu prendre une certaine liberté et traduire respectivement, dans l'esprit de Jonas, par «crainte désintéressée » et " peu égoïste ».)

Ces oscillations sont difficiles à comprendre. Certes, la traduction n'est jamais une chose aisée et on est souvent obligé de recourir à des procédés indirects à l'encontre de l'exigence d'une stricte littéralité. Il peut être approprié de varier la traduction d'un 
terme donné en fonction du contexte. Mais en ce qui concerne le problème indiqué - faire comprendre le sens de Heuristik der Furcht - ces oscillations ne facilitent pas la compréhension; elles empêchent d'apercevoir la cohérence de la trame verbale de l'ouvrage. Ces remarques critiques ne mettent pas en question la qualité de la traduction de Jean Greisch qui est globalement excellente. Il suffirait d'en effectuer une version révisée.

\section{La duplication de l'excellence : une critique ontologique}

Après ce long commentaire de la "critique existentielle» et de son corollaire, l'heuristique de la crainte, je reviens sur un thème abordé d'abord par Jonas et que j'avais laissé de côté. Leon Kass avait dressé un « pense-bête » (laundry list), une énumération des différents motifs possibles pour lesquels on peut souhaiter d'entreprendre le clonage. On y trouve pêle-mêle des motifs vulgaires, comme une fabrication à la chaîne d'êtres humains pour des besoins pratiques, mais aussi des motifs plus nobles comme la duplication d'êtres exceptionnels, doués d'un grand génie ou d'une grande beauté, ou la production d'un enfant ayant un génotype choisi à l'avance : d'une célébrité admirée, d'un chef défunt, du conjoint ou de soi-même ${ }^{16}$. Jonas retient, comme cible de sa critique, le motif le plus noble et non le plus trivial, car il procède de nos désirs les plus humanistes et les plus philanthropiques : ce qu'il appelle la duplication de l'excellence et qui, comme les autres techniques biogénétiques, procède d'un certain souhait ou désir (Wunsch) ${ }^{17}$. Avant d'introduire l'argument qu'il appelle la "critique existentielle » (que j'ai examiné plus haut), Jonas esquisse une interrogation portant sur l'ambition même qui nourrit le projet de reproduire et multiplier un grand artiste ou un grand savant. Qu'est-ce que nous recherchons et désirons au juste lorsque nous concevons un tel désir?

Jonas effectue là ce que j'appelle une critique du désir ou une critique de la faculté désirante, comportant deux volets: une interrogation réflexive sur les désirs qui inspirent nos projets, et une interrogation sur la désirabilité même de ces désirs.

L'argument de l'excellence, quoique naïf, n'est pas frivole dans la mesure où il fait appel à notre révérence de la grandeur et paie à celle-ci le tribut du désir que la race humaine s'orne de davantage de Mozarts, d'Einsteins ou de Schweizers. Soit dit en passant, personne ne cite, dans ce contexte, Nietzsche ou Kafka, et rares sont ceux qui citent Beethoven ou Michel-Ange - une preuve de l'eudémonisme secret de toute la rêverie : on voudrait avoir son génie heureux ou au moins joyeux. (TME, 186)

47 Ainsi, pourquoi ce projet que nous avons de multiplier les Mozart, Einstein ou Schweizer ? Je dis bien "nous ", car ce n'est pas l'un ou l'autre savant fou dans son laboratoire qui entretient de tels fantasmes; nous sommes tous, potentiellement, des docteurs Frankenstein prêts à tout pour réaliser nos rêves les plus élevés. Si certaines technologies se développent, ce n'est pas seulement en raison d'une dynamique propre de la technique, c'est aussi parce qu'elles peuvent faire fond sur l'existence chez les hommes de certains désirs (comme le fameux « désir d'enfant »). Jonas, à propos de la duplication de l'excellence, pose deux questions, correspondant à deux mises en évidence d'un désir sous-jacent. La première: pourquoi souhaiter avoir davantage de Mozart, etc.? La seconde : pourquoi cette préférence accordée à Mozart, et pas à Nietzsche ou à Kafka, ni même à Beethoven ou à Michel-Ange ? 
48 Envisagé sous ces deux angles, il apparaît que le projet de clonage repose sur une méconnaissance de la nature propre de la "génialité » ou de l'« excellence » humaine. D’une part, elle est par essence imprévisible et exceptionnelle et c'est méconnaître sa nature que de vouloir la fixer et la dupliquer. Elle comporte une contingence, quelque chose qui «ne devait pas être », et qui constitue précisément un miracle. Et d'autre part, elle comporte un revers - la peine, la souffrance, la douleur - : le génie doit accepter de peiner sur son œuvre (Beethoven et Michel-Ange comme emblèmes), d'être différent des autres, d'être malheureux ou névrosé ou malade (Nietzsche et Kafka comme emblèmes). Certes, il ne faudrait pas non plus, comme cela a été fait, lier de manière absolue la création et la folie ou le crime. Il n'en reste pas moins qu'elle a une part d'ombre, dont le projet de clonage ne veut rien entendre : on veut avoir des génies heureux.

Ces deux méconnaissances sont deux volets d'une même exclusion/forclusion de l'Autre, à savoir la visée du génie à l'état pur, sans reste ${ }^{18}$. Ce qui rejoint la critique existentielle : l'œuvre exceptionnelle est un novum, elle n'est pas l'émanation d'un " génie ", et le génie se découvre génie. Mozart se découvre Mozart, Einstein se découvre Einstein (et il est probable qu'il ne se représentait pas lui-même comme " génie »). Cet aspect, qui constitue un thème permanent de sa pensée, Jonas l'a mis en évidence sans vraiment le thématiser. Je propose d'appeler son argumentation une critique ontologique du clonage des génies. Elle est « ontologique ", parce qu'il s'agit du rapport de l'être et du non-être, et de la prise en considération (ou du déni) de la contingence inhérente à l'événement contingent qu'est l'excellence. Et ce, pour autant, bien sûr, qu'on ne nie pas tout simplement qu'existe une telle chose que l'excellence ou la génialité, en une attitude de dénégation ou de ressentiment.

Cet exemple du génie, ou disons plutôt, de l'excellence, de l'exceptionnalité, introduit directement à la « critique existentielle » : l'œuvre dite grande, géniale, exceptionnelle voire tout simplement bonne - n'est pas donnée comme telle à l'avance. Elle surgit de manière certes voulue, mais en même temps non maîtrisée, elle est un événement. Mis à part certains cas particuliers, l'auteur - artiste, savant - ne sait pas à l'avance quelle découverte il va faire ${ }^{19}$, comme il ne sait pas non plus à l'avance qu'il va être un être exceptionnel (même si les Muses ont pu veiller sur son berceau dès sa naissance). Il ne sait pas à l'avance qui il est ni qui il sera.

Du reste, cette ignorance n'est pas seulement le lot de ce qu'il est convenu d'appeler le "génie » ou les "génies"; elle est une caractéristique de la condition humaine en général, ou de l'existence comprise au sens fort. Cela montre que ce qu'il est convenu d'appeler un " génie » - une personnalité exceptionnelle, un être d'exception, moins par ses qualités innées que par les prouesses qu'il a accomplies dans son existence, par l'« excellence » qu'elle a manifestée - demeure ancré dans l'existence humaine telle qu'elle se déroule chez l'homme du commun, dans l'humanité commune. Car, comme l'a souligné Jonas, toute vie humaine, ne révèle ce qu'elle est qu'au cours de son existence.

Elle se découvre elle-même ou, ce qui revient au même, elle a la révélation de l'excellence de son œuvre ou de ses découvertes - au même titre qu'un humble artisan qui découvre son talent et sa vocation dans l'exercice même de son métier, au même titre qu'un soldat qui, à la guerre, se révèle à lui-même et aux autres comme courageux ou comme lâche, au même titre qu'un homme ou une femme qui, sans le savoir à l'avance, s'aperçoivent qu'ils sont un bon père ou une bonne mère. Car ni le génie ni la prouesse ou l'œuvre géniale ne sont préétablis. 
Il est écrit dans la Genèse : «Et Dieu créa l'homme à son image, à son image il le créa, il le
créa comme homme et femme », mais Goethe, dit Jonas, nous fait entendre comment
aujourd'hui l'art humain peut ambitionner «d'améliorer et de surpasser l'ouvrage du
Créateur ». Il cite alors un long passage de la scène du laboratoire où Wagner commente
pour Méphisto l'expérience qu'il est en train de réaliser : la fabrication de l'homoncule,
un petit homme qui grandit dans un bocal et ne va pas tarder à prendre la parole. Dans ce

Il est écrit dans la Genèse : «Et Dieu créa l'homme à son image, à son image il le créa, il le
créa comme homme et femme », mais Goethe, dit Jonas, nous fait entendre comment
aujourd'hui l'art humain peut ambitionner «d'améliorer et de surpasser l'ouvrage du
Créateur ». Il cite alors un long passage de la scène du laboratoire où Wagner commente
pour Méphisto l'expérience qu'il est en train de réaliser : la fabrication de l'homoncule,
un petit homme qui grandit dans un bocal et ne va pas tarder à prendre la parole. Dans ce

Il est écrit dans la Genèse : «Et Dieu créa l'homme à son image, à son image il le créa, il le
créa comme homme et femme », mais Goethe, dit Jonas, nous fait entendre comment
aujourd'hui l'art humain peut ambitionner «d'améliorer et de surpasser l'ouvrage du
Créateur ». Il cite alors un long passage de la scène du laboratoire où Wagner commente
pour Méphisto l'expérience qu'il est en train de réaliser : la fabrication de l'homoncule,
un petit homme qui grandit dans un bocal et ne va pas tarder à prendre la parole. Dans ce

Il est écrit dans la Genèse : «Et Dieu créa l'homme à son image, à son image il le créa, il le
créa comme homme et femme », mais Goethe, dit Jonas, nous fait entendre comment
aujourd'hui l'art humain peut ambitionner «d'améliorer et de surpasser l'ouvrage du
Créateur ». Il cite alors un long passage de la scène du laboratoire où Wagner commente
pour Méphisto l'expérience qu'il est en train de réaliser : la fabrication de l'homoncule,
un petit homme qui grandit dans un bocal et ne va pas tarder à prendre la parole. Dans ce

Il est écrit dans la Genèse : «Et Dieu créa l'homme à son image, à son image il le créa, il le
créa comme homme et femme », mais Goethe, dit Jonas, nous fait entendre comment
aujourd'hui l'art humain peut ambitionner «d'améliorer et de surpasser l'ouvrage du
Créateur ». Il cite alors un long passage de la scène du laboratoire où Wagner commente
pour Méphisto l'expérience qu'il est en train de réaliser : la fabrication de l'homoncule,
un petit homme qui grandit dans un bocal et ne va pas tarder à prendre la parole. Dans ce sexuée. C'est avec l'émergence de celle-ci que s'est introduit une dimension d'imprévisibilité ou d'indétermination :

Le hasard: c'est la source productive de l'évolution des espèces. Le hasard : c'est, pour chaque engendrement sexué, la garantie que chaque individu, à sa naissance, soit unique et qu'aucun ne ressemble à un autre. Le hasard assure la surprise du toujours neuf ${ }^{20}$. (TME 212)

Il existe un enregistrement de la conférence sur le rôle créateur de l'homme où ressort à la fois le talent d'orateur de Jonas et ses références à Goethe. Jonas, qui connaissait par cœur des pans entiers de la littérature allemande comme de la littérature classique et s'en nourrissait constamment dans ses écrits, fait plusieurs allusions dans sa critique des utopies technologiques à des mythes et symboles provenant de l'œuvre de Goethe : la boîte de Pandore, l'apprenti sorcier et surtout la scène du laboratoire du Faust II. Cette référence à Goethe, comme celles relatives aux mythes grecs et à la Bible, caractérise en général sa réflexion sur la technique et, en particulier, sur la biotechnologie. C'est un trait permanent de son style philosophique : une dimension symbolique et mythique. 
texte admirable se détache un vers extraordinaire : «Mais nous voulons désormais nous rire du hasard ", qui exprime de manière condensée le projet prométhéen de remplacer les aléas de la procréation naturelle par la fabrication délibérée et planifiée :

- Wagner :

Il se fait un être humain.

- Méphisto :

Un être humain? Et quel couple amoureux

Avez-vous donc enfermé dans la cheminée?

- Wagner :

À Dieu ne plaise ! La procréation telle qu'elle était de mode

Nous la déclarons une vaine plaisanterie [...]

Tout cela est déchu maintenant de sa dignité.

Si l'animal continue à prendre plaisir à ces choses,

L'homme avec ses belles facultés

Doit désormais avoir une origine plus haute et plus pure. [...]

Une grande entreprise paraît folle au début ;

Mais nous voulons désormais nous rire du hasard,

Et le cerveau destiné à penser supérieurement

Sera créé désormais aussi par un penseur ${ }^{21}$. mise en scène exceptionnelle de Peter Stein en 2000. Ce fut le cas exemplaire d'un éclairage réciproque entre un contenu mythique intemporel et l'actualité du présent. La possibilité imminente du clonage humain donne une actualité saisissante à la scène du laboratoire; mais le génie poétique de Goethe, sa capacité à exprimer des questions profondes par des mots très simples éclaire notre présent et ouvre la voie à notre réflexion philosophique et théorique.

Et pourtant, qu'il me soit permis d'apporter la contradiction sur un point: dans son magnifique éloge du hasard - d'autant plus splendide qu'il se réfère à Goethe et à ce passage génial du Second Faust - Jonas ne va pas assez loin. En effet, comme toute la génétique et la biologie moléculaire, il comprend la fécondation comme le résultat d'une combinaison aléatoire, avec l'image d'un "coup de dés", bref d'une combinatoire. Je considère qu'il faut ajouter à cette dimension (que j'appelle contingente et non pas " aléatoire »), un autre aspect: la synthèse créatrice, la position d'une forme nouvelle, grâce à laquelle chaque être humain, depuis la fécondation, est une nouvelle création et un nouveau départ. C'est plutôt de cette manière qu'il faut comprendre le thème commun à Jonas et à Arendt de la natalité, du surgissement d'individus toujours nouveaux et surprenants. 


\section{Tableau comparatif}

\begin{tabular}{|c|c|}
\hline $\begin{array}{l}\text { Biological Engineering - A Preview } \\
\text { (Philosophical Essays) } \\
\text { Futuristic Modes }\end{array}$ & $\begin{array}{l}\text { Lasst uns einen Menschen klonieren } \\
\text { (Technik, Medizin und Ethik) } \\
\text { Futuristiche Methoden I }\end{array}$ \\
\hline $\begin{array}{l}\text { A. Cloning } \\
\text { 1. Was is cloning? } \\
\text { 2. Questions about cloning }\end{array}$ & $\begin{array}{l}\text { 6. Klonieren } \\
\text { a. Was ist Klonieren? } \\
\text { b. Fragen zur Klonierung }\end{array}$ \\
\hline $\begin{array}{l}\text { a. The physical outcome of cloning } \\
\text { b. Reasons of cloning } \\
\text { c. Replication of excellence examined }\end{array}$ & $\begin{array}{l}\text { i. Das physiche Ergebnis der Klonierung } \\
\text { ii. Gründe für Klonierung } \\
\text { iii. Replizierung der Vortrefflichkeit }\end{array}$ \\
\hline $\begin{array}{l}\text { 3. Existential critique (159-163) } \\
\text { a. contemporariness of identical twins } \\
\text { b. uniqueness (160) } \\
\text { c. non contemporariness and the right to } \\
\text { ignorance (160) } \\
\text { § p. } 161 \\
\text { d. Knowledge, ignorance and freedom }\end{array}$ & $\begin{array}{l}\text { c. Existentielle Kritik (187-194) } \\
\text { i. Die Gleichzeitigkeit identischer Zwillinge } \\
\text { <supprimé dernier } \$> \\
\text { <supprimé> } \\
\text { ii. Nicht-Gleichzeitigkeit und das Recht zum } \\
\text { Nichtwissen } \\
\text { <ajout : } 189-190 \text { :,Hier sei eine } \\
\begin{array}{l}\text { Zwischenbemerkung eingeschaltet> } \\
\text { iii. Verderbliches Wissen } \\
\text { iv. Wissen, Unwissen und Freiheit }\end{array}\end{array}$ \\
\hline B. DNA Architecture & Futuristische Methoden II \\
\hline
\end{tabular}

\section{NOTES}

2. Jonas, Technik, Medizin und Ethik. Praxis des Prinzips Verantwortung, Francfort-sur-le-Main, Suhrkamp, 1987, p. 9. Dorénavant cité TME.

3. Tout se passe comme si un destin poursuivait Jonas. Suite à l'échec cuisant représenté par le refus de l'éditeur américain de son grand livre systématique Organism and Freedom, il avait pris le parti, faisant contre mauvaise fortune bon cœur, de publier en 1966, sous le titre de The Phenomenon of Life, un ensemble d'études préparatoires, à la place de cet ouvrage systématique. En 1985, à la place du second volume du Principe responsabilité consacré à la casuistique, il publie, en raison de son grand âge, cet ensemble d'études fragmentaires.

4. En ce qui concerne les problèmes posés par la définition pragmatique de la mort: la «mort cérébrale " comme critère (déclaration de Harvard de 1969), la prise de position de Jonas, exprimée dès 1970, a été prolongée et complétée récemment par Laura Bossi dans Les frontières de 
la mort, Paris, Payot, 2012, où elle fait le bilan actuel de la question, d'un point de vue à la fois médical et philosophique.

5. Voir entre temps la traduction de ce texte, et du recueil auquel il appartient, sous le titre de "Biotechnologies. Une vision anticipatrice", in Essais philosophiques, D. Bazin et O. Depré éd., Paris, Vrin, 2013 ; ces Philosophical Essays seront dorénavant cité PhE suivi de la pagination qui figure aussi en marge de la traduction française.

6. Dans mes lectures philosophiques, je garde toujours un regard philologique, une attention non à la matière linguistique, mais aussi aux différentes variantes des textes. Un tel regard peut être un auxiliaire précieux de l'examen philosophique (même s'il ne doit pas prédominer) en permettant de suivre la genèse d'une pensée.

7. Voir, en annexe, une table comparative des deux versions où sont indiquées les quelques différences.

8. Leon R. Kass (né en 1939) relate les circonstances de leur rencontre dans « Appreciating The Phenomenon of Life ", in The Legacy of Hans Jonas, Hastings Center Report, vol. 25, $\mathrm{n}^{\circ}$ 7, Édition spéciale, 1995.

En 1998, Kass a publié The Ethics of Human Cloning. Dans le présent article je ne me réfèrerai pas directement aux écrits de Kass, mais uniquement aux citations qu'en donne Jonas.

9. Kass, "New Beginnings of Life ", in The New Genetics and the Future of Man, Michael P. Hamilton éd., Michigan, Grand Rapids, 1972, pp. 14-63.

10. PhE, 160, dans ma traduction comme pour tous les passages à venir de cet ouvrage. Je cite d'après la citation de Jonas, qui se réfère manifestement au texte de Kass.

11. Jonas emploie un terme qu'il ne commente pas : «ein Selbst », un Soi. Dans Soi-même comme un autre, Paul Ricœur distinguait entre mêmeté et ipséité, entre idem et ipse. L'argument de Leon Kass, le droit à l'unicité du génotype, est un droit à une différence du génotype par opposition à la mêmeté. L'argument de Jonas, avec la critique existentielle, est un droit au déploiement libre d'une ipséité.

12. Cette tension féconde entre essentialisme et existentialisme est l'un des traits de la pensée de Jonas.

13. Je songe au savoureux passage de Bergson sur « l'armoire des possibles ».

14. Jonas, Das Prinzip Verantwortung, Francfort-sur-le-Main, Suhrkamp, 1984, pp.63-64. Dorénavant cité PV. Le principe responsabilité, trad. J. Greisch, Paris, Cerf, 1990, p. 49, trad. mod. Dorénavant cité PR.

15. «Préservation de l'humanité et image de l'homme », Hans Jonas, Études Phénoménologiques, $\mathrm{n}$ ○ 8, Bruxelles, Ousia, 1988, pp. 33-68. Je l'appelle «princeps» parce qu'était le premier article paru en français sur la pensée de Jonas.

16. On peut s'étonner qu'il ne mentionne pas l'un des motifs les plus courants et évidents : le désir d'immortalité, qui sous-tend d'autres utopies technologiques, comme le projet d'hibernation du " mouvement cryonique ", lancé en 1964 par Richard Ettinger.

17. Ce thème du désir (Wunsch) est présent dans Le principe responsabilité, au paragraphe sur l'heuristique de la crainte, lorsque Jonas avance que nos craintes sont plus révélatrices que nos désirs pour savoir ce que nous apprécions vraiment. "Wunsch », c'est littéralement le « souhait » ou le « vœu », et cela rejoint l'emploi de cette notion chez Freud (notamment dans son analyse de l'illusion). La traduction par « désir » est justifiée.

18. Cela rejoint une méditation critique sur la beauté, qui ne peut exister à l'état pur. Je songe ici à la polémique de Gombrowicz « contre la poésie », dont il ressort que c'est la poésie pure ou l'art pur qui doit être mis en question, de la même façon, que le génie à l'état pur, sans aucune scorie. Je songe aussi à la réflexion critique de Philippe Muray.

19. Comme l'écrit Jonas, la recherche scientifique, ou plus exactement la découverte, n'est pas programmable, elle ne peut pas être « vorbudgetiert ». 
20. À cet égard, l'actuelle oblitération de la vie sexuée (c'est-à-dire divisée), dans la sensibilité contemporaine, va de pair avec une disparition tendancielle de l'individuation.

21. Cité par Jonas, TME, 211 sq., traduction - légèrement modifiée - de Henri Lichtenberger, le Second Faust, Acte II, scène 2, vers 6835-6870, Paris, Aubier-Montaigne, pp. 76-77. 